Britain has the most extensive survey network, measuring smoke and sulphur dioxide, organized by the Warren Spring Laboratory of the Ministry of Technology. Pollution data from most other countries are still insufficient.

Pollution may increase disastrously with the increase in world population and industrialization. But there are hopeful developments-fow countries now allow unlimited emissions of smoke (which may contain fluorine, sulphur and other poisons) even in remote rural areas. In Britain smoke pollution has decreased substantially in recent years, and sulphur dioxide levels have ceased to rise, despite increased use of fuels containing sulphur. Other countries are making attempts to solve their problems. This symposium showed that the ecologist may make as great a contribution as the engineer. Where pollution cannot be prevented at source, the planned use of the correct type of vegetation may have very useful effects.

\section{Amine Oxidase Isoenzymes}

\section{from our Medical Biochemistry Correspondent}

There is now evidence for the existence of more than one monoamine oxidase in mammalian tissues. The monoamine oxidases inactivate amines by oxidative deamination, and monoamine oxidase inhibitors are widely used in the treatment of depression, but the effects of increased amine concentrations on the blood pressure can lead to undesirable side-effects. The effect of these drugs is normally measured rather crudely by measuring the increase in urinary tryptamine, which is also affected by amines in the diet. In an attempt to estimate directly the effect of inhibitors on monoamine oxidase in the blood, Robinson, Lovenberg, Keiser and Sjoerdsma (Biochem. Pharmacol., 17,$109 ; 1968$ ) showed that there were great differences between the amine oxidase activities of platelets and plasma. The platelet enzyme, like the human liver amine oxidase, was inhibited by antidepressant drugs but not by the anti-tubercular drug isoniazid or potassium cyanide. The plasma enzyme was inhibited by isoniazid and potassium cyanide but was less sensitive to the antidepressant drugs than the liver and platelet enzymes. When the kinetics of the platelet enzyme were compared with those of the rat liver enzyme, both showed more activity with some substrates than with others, but the maximum velocities and substrate affinities differed considerably. When eighteen humans were given antidepressant drugs or isoniazid, the inhibition of platelet amine oxidase correlated well with the increase in urinary tryptamine excretion, but the inhibition of plasma amine oxidase did not.

The study of a new monoamine oxidase inhibitor, NSD 2023, has provided evidence of more than one amine oxidase in mouse tissues (Squires and Larssen, Biochem. Pharmacol., 17, 369; 1968). The drug $\gamma$-morpholino butyrophenone (NSD 2023) is an anticonvulsant and protects against the effects of reserpine, and also acts as an amine oxidase inhibitor at very low doses. The maximum inhibition in vivo in mouse brain was 40 per cent with kynuramine as substrate and 70 per cent using tryptamine as substrate. This suggests that mouse brain must contain at least two amine oxidases which differ in their susceptibility to inhibition by NSD 2023. The proportions in different tissues must vary, because both in vivo and in vitro the drug produced more inhibition in preparations from brain and kidney than from liver.

An interesting variation in activity of amine oxidase during the human menstrual cycle has recently been demonstrated (Southgate et al., Biochem. Pharmacol., 17, 721; 1968). Previous histochemical studies had shown that in the non-secretory and carly secretory phases of the menstrual cycle amine oxidase was present in granules in the cells of the endometrium, but in the late secretory phase there was diffuse staining in the cytoplasm as well. In this investigation normal endometrial biopsies from 30 women were stained histochemically for amine oxidase activity and the production of radioactive aldehyde from ${ }^{14} \mathrm{C}$-tryptamine was measured. The histochemical results confirmed the earlier observations, and the quantitative assays showed that there was six times as much activity in late secretory specimens as in specimens in the nonsecretory and early secretory phases of the menstrual cycle. They could not demonstrate any changes in activity during the oestrous cycle in the rat. If some of the monoamine oxidase activity in human brain is also hormone-dependent, this might be related to postpuerperal depression and variations in depression during the menstrual cycle. Further investigations of the differences in amine oxidase activities in different tissues might lead to drugs affecting some amine concentrations without the undesirable side-effects of present preparations.

\section{RI7 A Protein}

\section{from our Cell Biology Correspondent}

LAST year (see Nature, 216, 541; 1967) Roberts and Argetsinger-Steitz successfully reconstituted infective particles of the RNA bacteriophage $R 17$ and in so doing proved that the A protein is a structural component of the phage and essential for infectivity. The success of this reconstitution experiment depended on the isolation of the A protein and, in two papers in the current Journal of Molecular Biology (33, 923 and 937; 1968), Argetsinger-Steitz reports how the A protein was isolated and characterized and its aminoacid composition analysed. It is clear from this work that each $R 17$ phage contains only one molecule of the histidine containing A protein which has a molecular weight of about 38,000 .

To show that the A protein is a structural component of $R 17$ grown in vivo, Argetsinger-Steitz exploited the fact that the amino-acid histidine is absent from the phage coat protein. Phage was grown in the presence of labelled histidine and then isolated, purified and assayed for radioactivity. The phage contained histidine. Fractionation of the proteins isolated from the labelled phage by three distinct methods, polyacrylamide gel electrophoresis, ion exchange chromatography and filtration on 'Sephadex', all showed that the histidine was present in phage specific A protein. The behaviour of the A protein on 'Sephadex' indicates its molecular weight is about 35,000-40,000. Furthermore, as expected from the in vitro reconstitution experiment, A protein is totally absent from the defective particles produced by phage which have an amber mutation in the $\mathrm{A}$ cistron.

Since the A protein has a molecular weight of 35,000 40,000 , it must contain about 350 amino-acids; $R 17$ coat protein contains 129 amino-acids, and Capechi 